\title{
Neuropeptide Y upregulates Runx2 and osterix and enhances osteogenesis in mouse MC3T3-E1 cells via an autocrine mechanism
}

\author{
BO ZHANG ${ }^{1}$, XIAOLEI ZHANG ${ }^{2}$, JUAN XIAO $^{3}$, XUGUANG ZHOU $^{1}$, YUAN CHEN $^{4}$ and CHUNZHENG GAO $^{5}$ \\ ${ }^{1}$ Department of Joint Surgery, The Second Hospital, Cheeloo College of Medicine, Shandong University, Jinan, \\ Shandong 250033; ${ }^{2}$ Department of Obstetrics and Gynecology, Qilu Hospital, Cheeloo College of Medicine, \\ Shandong University, Jinan, Shandong 250012; ${ }^{3}$ Department of Evidence-Based Medicine, \\ Institute of Medical Sciences, The Second Hospital, Cheeloo College of Medicine, Shandong University; \\ Departments of ${ }^{4}$ Central Research Lab and ${ }^{5}$ Spinal Surgery, The Second Hospital, \\ Cheeloo College of Medicine, Shandong University, Jinan, Shandong 250033, P.R. China
}

Received February 5, 2020; Accepted August 20, 2020

DOI: $10.3892 / \mathrm{mmr} .2020 .11506$

\begin{abstract}
The neuropeptide Y (NPY) system is considered one of the primary neural signaling pathways. NPY, produced by osteoblasts and other peripheral tissues, is known to inhibit biological functions of osteoblasts. However, until recently, little was known of the autocrine mechanism by which NPY is regulated. To investigate this mechanism, overexpression plasmids and small interfering RNA (siRNA) targeting NPY were transfected into the MC3T3-E1 cell line to observe its effects on osteogenesis. NPY overexpression was found to markedly enhance the osteogenic ability of MC3T3-E1 cells by an autocrine mechanism, coincident with the upregulation of osterix and runt-related transcription factor 2 (Runx2). Furthermore, NPY increased the activities of alkaline phosphatase (ALP) and osteocalcin (OCN) by upregulating their osteoblastic expression in vitro (as well as that of osterix and Runx2). Following transfection with NPY-siRNA, the osteoblastic ability of MC3T3-E1 cells was markedly decreased, and NPY deficiency inhibited the protein expression of osterix, Runx2, OCN and ALP in primary osteoblasts. Collectively, these results indicated that NPY played an important role in osteoblast differentiation by regulating the osterix and Runx 2 pathways.
\end{abstract}

\section{Introduction}

Severe trauma, tumor resection, cancer or congenital diseases can result in large segmental bone defects (1). Synthetic bone

Correspondence to: Professor Chunzheng Gao, Department of Spinal Surgery, The Second Hospital, Cheeloo College of Medicine, Shandong University, 247 Beiyuan Street, Jinan, Shandong 250033, P.R. China

E-mail: gaochunzheng1964@sina.com

Key words: neuropeptide Y, MC3T3-E1 cell line, runt-related transcription factor 2, osterix, small interfering RNA graft substitutes combined with bioactive molecules, such as growth factors, peptides and small molecules, can improve the delivery of bone precursor cells, as well as subsequent bone formation and metabolism (2). Bioactive molecules are required in patients with larger bone defects $(>2 \mathrm{~cm})(2)$. For this purpose, growth factors, peptides and small molecules are currently being evaluated at the pre-clinical and clinical levels (2). Based on previous findings, combining Neuropeptide Y (NPY) with the appropriate corresponding bone scaffold may bring about effective clinical translations and novel insights into the future of bone transplantation.

NPY, a 36-amino acid peptide, is a neurotransmitter located in the brain and autonomic nervous system (3). As a typical regulator of appetite and energy balance, NPY serves an important role in the central nervous system (4), as well as a protective role in chronic bone loss-induced stress (5). NPY, which is stored and released together with noradrenaline in the process of nerve stimulation, is part of the sympathetic arm of the peripheral nervous system (4). NPY has been identified in several cell types, including osteoblasts and adipocytes (6-8), and has previously been demonstrated to regulate the balance of bone mass in both the central and peripheral regions $(9,10)$.

NPY receptors are members of the $G$ protein-coupled receptor superfamily, and five subtypes (Y1, 2, 4, 5 and 6) are currently acknowledged in humans (11). However, in rodents, bone mass is regulated by the Y1 and Y2 receptors only (12). In previous studies, inhibiting the Y1 receptor increased the mRNA expression levels of osterix and runt-related transcription factor 2 (Runx2), two transcription factors essential for osteoblastic differentiation and bone formation (13). Y1 inhibition can also promote the activity of Runx2 and osterix, and thus may contribute to osteoblastic differentiation and the expression of osteocalcin (OCN), alkaline phosphatase (ALP), bone sialoprotein (BSP) and collagen 1 $\alpha$ (14). However, whether NPY itself is able to modulate osteoblasts via Runx2, osterix and bone morphogenetic protein (BMP) signaling remains to be elucidated.

Igwe et al (15) revealed that NPY is expressed in osteoblasts and serves an important role in osteogenic differentiation. They 
also identified higher NPY mRNA expression in bone fractions highly abundant in mature osteoblasts and osteocytes. In a previous study, treatment of osteoblasts with NPY decreased the expression of markers of osteoblast differentiation (9). Collectively, the aforementioned studies focused on cellular proliferation and osteogenesis as a result of the exogenous administration of NPY. However, little is known of the effects of NPY alterations at the genetic level. In the present study, the aim of the study was to investigate the effects of regulating NPY by an autocrine mechanism (coincident with the regulation of Runx2 and osterix) using the MC3T3-E1 cell line.

In a previous report, non-tumorous MC3T3-E1 cells were extracted from the skull and underwent a typical osteoblastic differentiation process in vitro (16). In the present study, experiments were conducted by transfecting MC3T3-E1 cells with NPY small interfering RNA (siRNA) or overexpression plasmids to alter its expression level; this method demonstrated the autocrine, rather than the exocrine mechanism by which NPY regulates osteoblastic function. The osteoblastic ability of MC3T3-E1 cells was significantly enhanced following NPY overexpression, resulting in an increase in the expression of ALP, OCN, Runx 2 and osterix mRNA levels at different time points, compared with those in untreated cells. In conclusion, this study demonstrated that NPY signaling serves a positive role in osteogenic differentiation of MC3T3-E1 cells by upregulating Runx 2 and osterix in vitro. This will help to solve the clinical problem of large bone defects caused by severe trauma, tumor resection, cancer or congenital diseases in the future.

\section{Materials and methods}

Cell isolation, culture and treatment. The MC3T3-E1 cell line was purchased from the Cell Bank of Union Medical University (http://sbm.pumc.edu.cn/). MC3T3-E1 cells were cultured in $\alpha$-minimum essential medium (MEM with $10 \%$ Fetal Bovine Serum, both Gibco; Thermo Fisher Scientific, Inc.) supplemented with $10 \mathrm{mmol} \beta$-glycerophosphate, $50 \mu \mathrm{g} / \mathrm{ml}$ ascorbic acid and $10 \mathrm{mmol}$ dexamethasone, and maintained at $37^{\circ} \mathrm{C}$ in $5 \% \mathrm{CO}_{2}$. The culture medium was renewed every 2-3 days.

Expression plasmids and transfection. MC3T3-E1 cells $\left(2.0 \times 10^{6}\right.$ cells/well $)$ were seeded into 6 -well plates $(2 \mathrm{ml} /$ well $)$ and cultured at $37^{\circ} \mathrm{C}\left(5 \% \mathrm{CO}_{2}\right)$ until $70-80 \%$ confluence. In order to study the subsequent biological effects of NPY on MC3T3-E1 cells, three different groups of siRNA (final concentration: $10 \mathrm{nM}$ ) and an NPY overexpression plasmid (final concentration: $25 \mathrm{nM}$ ) were designed by Wuhan Qingke Innovation Biotech Co., Ltd., along with a scrambled siRNA group (Wuhan Qingke Innovation Biotech Co., Ltd.) and an empty vector group (Wuhan Qingke Innovation Biotech Co., Ltd.), which acted as the negative controls. The three NPY siRNA target sequences (siRNA1, siRNA2 and siRNA3) are displayed in Table I. Then, untransfected cells were used as the control group for subsequent experiments. The cells were transfected with NPY siRNA and overexpression plasmids; NPY siRNA transfection was conducted for $48 \mathrm{~h}$ using RFect siRNA transfection reagent (Changzhou Baidai Biotechnology Co., Ltd.), and the NPY overexpression plasmid was transfected using RFect DNA transfection reagent (Changzhou Baidai
Biotechnology Co., Ltd.), also for $48 \mathrm{~h}$. Material synthesis and reagent preparation used in RFect siRNA and plasmid DNA transfection were performed according to the instructions of the transfection kits. Statistical analysis results showed that NPY siRNA3 had the highest interference efficiency, and as a result, siRNA3 was used for subsequent experimentation. The cells were then cultured for 7 days in the same conditions as aforementioned.

Reverse transcription-quantitative $(R T-q) P C R$ analysis. At 4 and 7 days post-transfection, MC3T3-E1 cells were collected and gene expression was detected by RT-qPCR. Total RNA was isolated using TRIzol ${ }^{\circledR}$ reagent (Tiangen Biotech Co., Ltd.) and quantified using the NanoDrop ${ }^{\mathrm{TM}} 2000$ (Thermo Fisher Scientific, Inc.). The RNA was reverse transcribed into cDNA using the RevertAid First Strand cDNA Synthesis kit (Thermo Fisher Scientific, Inc.), according to the manufacturer's protocol; the primer sequences for NPY, Runx2, OCN, ALP, osterix and GADPH (Wuhan Qingke Innovation Biotechnology Co., Ltd.) are displayed in Table I. The FastStart Universal SYBR-Green Master (Rox) was then used to perform qPCR with the LightCycler ${ }^{\circledR} 480$ Instrument II system (both Roche Diagnostics), under the following thermocycling conditions: $95^{\circ} \mathrm{C}$ for $5 \mathrm{~min}, 44$ cycles at $95^{\circ} \mathrm{C}$ for $15 \mathrm{sec}$, and then $72^{\circ} \mathrm{C}$ for $20 \mathrm{sec}$. Melting curve analysis was used to confirm the specificity of transcription amplification. Fold changes in target gene expression were quantified using the $2^{-\Delta \Delta \mathrm{Cq}}$ method (17) after normalization to the internal reference gene (GAPDH).

Western blot analysis. The cells were harvested and lysed with RIPA lysis buffer containing phenylmethane sulfonyl fluoride and protease inhibitors (both Sigma-Aldrich; Merck $\mathrm{KGaA}$ ). The concentration of each sample was determined by a bicinchoninic acid protein assay. Proteins from each group (30 $\mu \mathrm{g} /$ lane) were resolved via SDS-PAGE on a $10 \%$ gel, and subsequently transferred to PVDF membranes. After blocking with 5\% BSA (Sigma-Aldrich; Merck KGaA) at room temperature for $1 \mathrm{~h}$ in a phosphate buffered solution (PBS with $0.1 \%$ Tween-20; $\mathrm{pH} 7.5$ ) and washing in TBS with $0.05 \%$ Tween-20 (TBST), the membranes were incubated with anti-NPY (1:1,000; cat. no. DF6431; Affinity Biosciences), anti-OCN (1:500; cat. no. PAA471Mu01; Cloud-Clone Corp.), anti-ALP (1:1,500; cat. no. 11187-1-AP; ProteinTech Group, Inc.), anti-osterix (1:1,000; cat. no. bs-1110R; BIOSS) and anti-Runx2 (1:500; cat. no. PAB011Mu01; Cloud-Clone Corp.) antibodies overnight at $4^{\circ} \mathrm{C}$. After rinsing with TBST, the membranes were all incubated with a secondary antibody (horseradish peroxidase-linked guinea pig anti-rabbit IgG polyclonal; 1:2,000; cat. no. SAA544Rb59; Cloud-Clone Corp.) at $4^{\circ} \mathrm{C}$ for $1 \mathrm{~h}$, and then developed using a chemiluminescence detection system (Pierce; Thermo Fisher Scientific, Inc.). The data were analyzed using ImageJ software (Version 1.8.0; National Institutes of Health); the relative protein expression level was calculated as the grey value ratio of the target protein to GAPDH.

Statistical analysis. The experimental data are presented as the mean \pm SD of three independently performed experiments. One-way ANOVA was used to analyze the overall differences 
Table I. Primers used for reverse transcription-quantitative PCR.

\begin{tabular}{|c|c|c|}
\hline Gene name & Primer sequences $\left(5^{\prime} \rightarrow 3^{\prime}\right)$ & GenBank number \\
\hline NPY & $\begin{array}{l}\text { Sense: CTCGTGTGTTTGGGCATTC } \\
\text { Antisense: TAGTGTCGCAGAGCGGAGTA }\end{array}$ & NM_023456 \\
\hline siRNA1 & $\begin{array}{l}\text { Sense: CCAAACAAUAGACAUGCUUdTdT } \\
\text { Antisense: AAGCAUGUCUAUUGUUUGGdTdT }\end{array}$ & NM_000909.6 \\
\hline siRNA2 & $\begin{array}{l}\text { Sense: GCCCUUUGUGAUCUAUCAAdTdT } \\
\text { Antisense: UUGAUAGAUCACAAAGGGCdTdT }\end{array}$ & NM_000909.6 \\
\hline siRNA3 & $\begin{array}{l}\text { Sense: CCAAGCGAAUCAACAUCAUdTdT } \\
\text { Antisense: AUGAUGUUGAUUCGCUUGGdTdT }\end{array}$ & NM_000909.6 \\
\hline Runx2 & $\begin{array}{l}\text { Sense: GACGAGGCAAGAGTTTCACC } \\
\text { Antisense: GGACCGTCCACTGTCACTTT }\end{array}$ & NM_009820 \\
\hline $\mathrm{OCN}$ & $\begin{array}{l}\text { Sense: CAAGCAGGGAGGCAATAAGG } \\
\text { Antisense: CGTCACAAGCAGGGTTAAGC }\end{array}$ & NM_007541 \\
\hline GAPDH & $\begin{array}{l}\text { Sense: TCCACCACCCTGTTGCTGTA } \\
\text { Antisense: ACCACAGTCCATGCCATCAC }\end{array}$ & NM_002046.3 \\
\hline ALP & $\begin{array}{l}\text { Sense: TGGTCACAGCAGTTGGTAGC } \\
\text { Antisense: CTGAGATTCGTCCCTCGCTG }\end{array}$ & NM_001287172.1 \\
\hline Osterix & $\begin{array}{l}\text { Sense: GATGGCGTCCTCTCTGCTTG } \\
\text { Antisense: TCTTTGTGCCTCCTTTCCCC }\end{array}$ & NM_130458.3 \\
\hline
\end{tabular}

NPY, neuropeptide Y; Runx2, runt-related transcription factor 2; OCN, osteocalcin; ALP, alkaline phosphatase; GAPDH, glyceraldehyde-3-phosphate dehydrogenase; siRNA, small interfering RNA.

between multiple groups. The Scheffe (Fig. 1A and B) or least significant difference post hoc tests were used to evaluate the differences between groups using SPSS 18.0 software (SPSS, Inc.); representative bar graphs were created in GraphPad Prism v.6.0 (GraphPad Software, Inc.). $\mathrm{P}<0.05$ was considered to indicate a statistically significant difference.

\section{Results}

Screening of NPY-specific siRNA. Comparison of the control, siRNA1, siRNA2 and siRNA3 groups was conducted by one-way ANOVA followed by Scheffe post hoc test, and the differences among the groups were statistically significant $(\mathrm{P}<0.01$ or $\mathrm{P}<0.001)$. NPY siRNA3 had the highest interference efficiency of $>75 \%$, and as a result, siRNA3 was used for subsequent experimentation (Fig. 1A).

Secretion and expression of NPY in MC3T3-E1 cells. First, NPY secretion from MC3T3-E1 cells was verified at 4 and 7 days; NPY was consistently expressed at all time points, which provided the basis for the following experiments (Fig. 1B). In addition, NPY mRNA was sustainably expressed in both the experimental and control groups (Fig. 1B). Furthermore, the overexpression plasmids significantly promoted mRNA expression in MC3T3-E1 cells, which was increased by $\sim 3.9$-fold at day 4 and 4.3-fold at day 7, in comparison with the empty vector group $(\mathrm{P}<0.001$; Fig. $1 \mathrm{~B})$.

In order to more fully understand the role of NPY, MC3T3-E1 cells were transfected with NPY siRNA and cultured for $48 \mathrm{~h}$, and changes in NPY mRNA expression were observed over time. NPY mRNA expression was decreased by $\sim 0.75$-fold at day 4 and $\sim 0.55$-fold at day 7 , compared with the control and scrambled siRNA groups; there was statistical significance at day $4(\mathrm{P}<0.05$; Fig. $1 \mathrm{~B})$ and no statistical significance at day 7. Conversely, semi-quantitative analysis revealed that NPY overexpression increased NPY protein expression by 1.2 -fold at day 4 and 1.5 -fold at day 7 , compared with the control group ( $\mathrm{P}<0.05$; Fig. $1 \mathrm{C}$ and $\mathrm{D})$. NPY siRNA was then used to further elucidate the role of NPY in MC3T3-E1 cells. NPY protein expression was decreased by $\sim 0.45$-fold and 0.35 -fold at days 4 and 7 post-transfection, respectively $(\mathrm{P}<0.05$; Fig. 1C and D).

NPY is critical for osteoblastic differentiation. The expression levels of bone-formation factors, such as ALP and OCN, are determined by the different stages of bone differentiation (18). In the present study, ALP and OCN mRNA was consistently expressed in the three siRNA groups at days 4 and 7 post-transfection (Fig. 2A and B). The effects of NPY overexpression were then investigated using the MC3T3-E1 cell line, and NPY overexpression was found to significantly increase ALP mRNA expression by $~ 3.7$ - and 4.9-fold at days 4 and 7, and OCN mRNA expression by $\sim 4.0$ - and 4.3-fold at days 4 and 7 , respectively, compared with the control group $(\mathrm{P}<0.001$; Fig. 2A and B).

To further determine the effects of NPY on ALP and OCN mRNA , MC3T3-E1 cells were transfected with NPY siRNA for $48 \mathrm{~h}$, and the effects were observed over a subsequent time period. At days 4 and 7, ALP mRNA was decreased by $\sim 0.56$ - and 0.26 -fold, and OCN mRNA was decreased 

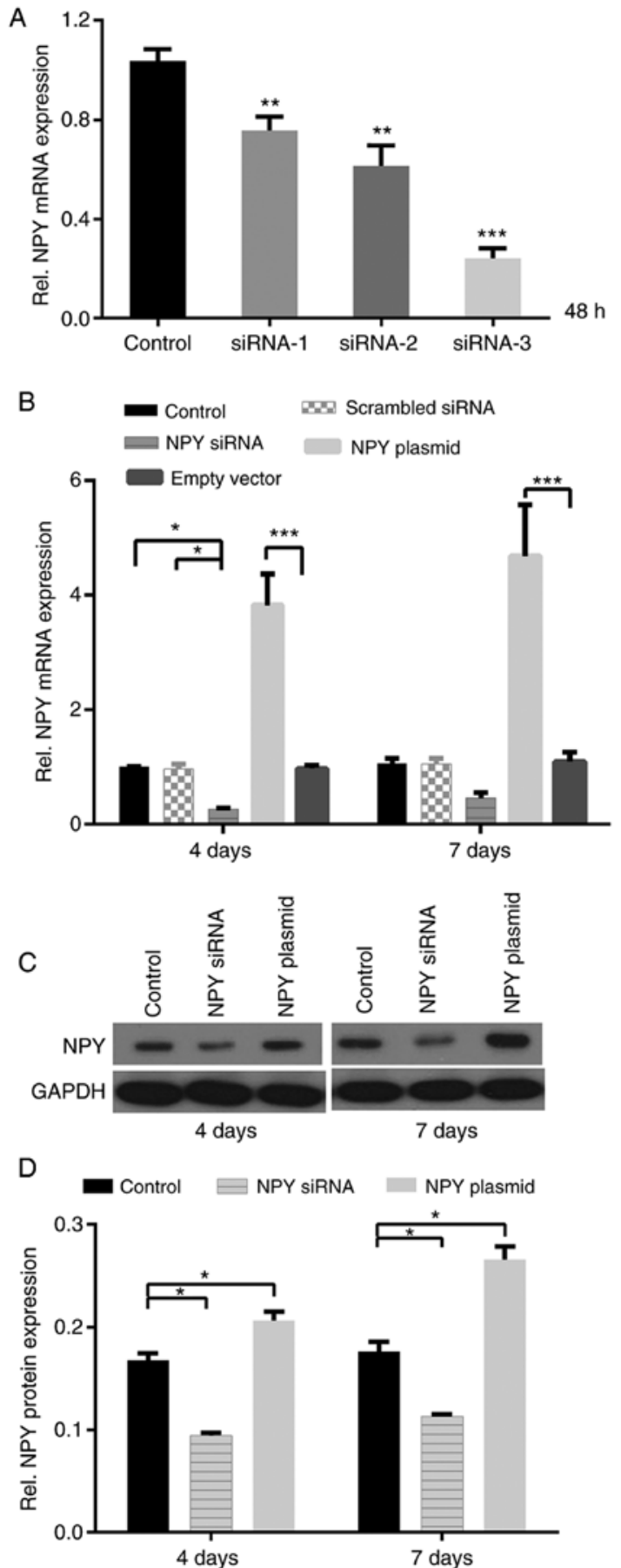

Figure 1. Expression of NPY mRNA and protein in MC3T3-E1 cells. (A) Effects of siRNA inhibition on the expression of NPY mRNA at $48 \mathrm{~h}$ ${ }^{* *} \mathrm{P}<0.01,{ }^{* * *} \mathrm{P}<0.001$ vs. Control. NPY (B) mRNA expression at days 4 and 7. NPY protein expression at days 4 and 7 (C) evaluated by western blotting and (D) semi-quantified. ${ }^{*} \mathrm{P}<0.05,{ }^{* * *} \mathrm{P}<0.001$. NPY, neuropeptide $\mathrm{Y}$; siRNA, small interfering RNA.

by $\sim 0.60$ - and 0.35 -fold, respectively, compared with the control group. The differences were statistically significant at day 4 ( $\mathrm{P}<0.05$; Fig. $2 \mathrm{~A}$ and $\mathrm{B})$. The ALP mRNA were also compared between the siRNA and overexpression groups, and overexpression was found to increase these levels $\sim 7.9$-fold (compared with siRNA inhibition) at day 4 and $\sim 6.5$-fold at day 7 , and OCN mRNA levels $~ 10.1$-fold (compared with siRNA inhibition) at day 4 and $\sim 6.7$-fold at day 7 , respectively $(\mathrm{P}<0.001$; Fig. 2A and B).
Semi-quantitative analysis of protein expression revealed that OCN protein expression was increased by 1.4-fold at day 4, and 1.6-fold at day 7 following NPY overexpression, compared with the control group; ALP protein expression was increased by 1.6 -fold at days 4 and 7 (P<0.05; Fig. 3A-C).

Following NPY siRNA interference, ALP and OCN protein expression was also assessed; the level of ALP at days 4 and 7 was decreased by $\sim 0.60$-fold, and the level of OCN protein expression was decreased by $\sim 0.64$-fold at day 4 and $\sim 0.78$-fold at day 7 , compared with the control group $(\mathrm{P}<0.05$; Fig. 3A-C).

$N P Y$ regulates Runx 2 and osterix expression in MC3T3-E1 cells. To the best of our knowledge, Runx 2 is an important early transcription factor involved in the expression of osteoblast-specific genes, and the differentiation of mesenchymal stem cells into osteoblasts (19). In addition, Runx2 is hypothesized to serve a primary role in the terminal differentiation of osteoblasts (20).

Therefore in the present study, the effects of NPY on Runx2 and osterix expression were determined using MC3T3-E1 cells. NPY overexpression was found to significantly increase Runx 2 and osterix mRNA expression. Compared with the control group, Runx 2 mRNA expression increased by $\sim 5$.4-fold at day 4 , and $\sim 6$-fold at day 7 . Additionally, osterix mRNA was increased by $\sim 2.7$-fold at day 4 and $\sim 3$-fold at day 7, compared with the control group. Of note, the level of Runx 2 mRNA expression was twice that of osterix $(\mathrm{P}<0.001$; Fig. $2 \mathrm{C}$ and $\mathrm{D}$ ).

To further understand the effects of NPY on Runx2 and osterix mRNA expression, MC3T3-E1 cells were transfected with NPY siRNA and the expression levels of Runx2 and osterix mRNA were determined at subsequent time points. At day 4 , Runx 2 mRNA expression was decreased by $\sim 0.50$-fold compared with the control group, and $\sim 0.35$-fold at day 7 . Osterix mRNA expression decreased by $\sim 0.80$-fold at day 4 and 0.55 -fold at day 7 , compared with the control group. The differences in osterix mRNA expression were statistically significant at day $4(\mathrm{P}<0.001$; Fig. $2 \mathrm{C}$ and $\mathrm{D})$.

Next, the levels of Runx 2 and osterix mRNA expression were compared between the siRNA and overexpression groups. In the overexpression group, Runx 2 mRNA expression was 10-fold higher than that of the siRNA group at days 4 and 7, and osterix mRNA expression was $\sim 15$-fold greater than that observed in the siRNA group at day 4 , and $\sim 8$-fold higher at day 7 ( $\mathrm{P}<0.001$; Fig. $2 \mathrm{C}$ and $\mathrm{D})$.

Semi-quantitative analysis of Runx 2 and osterix expression revealed that compared with the control group, NPY overexpression increased Runx 2 protein expression by 1.6-fold at day 4, and 1.2-fold at day 7. Similarly, osterix protein expression was increased by 1.6 -fold at day 4 , and 2.1-fold at day 7, compared with the control group $(\mathrm{P}<0.05$; Fig. 3A-C).

In the siRNA group, Runx 2 protein expression was decreased by $\sim 0.40$ - and 0.55 -fold at days 4 and 7 , respectively, compared with the control group. There was no statistical difference in Runx 2 mRNA expression in the siRNA group in Fig. 2A, whereas the difference in Runx 2 protein expression was statistically significant in Fig. 3A-C $(\mathrm{P}<0.05)$. Likewise, 

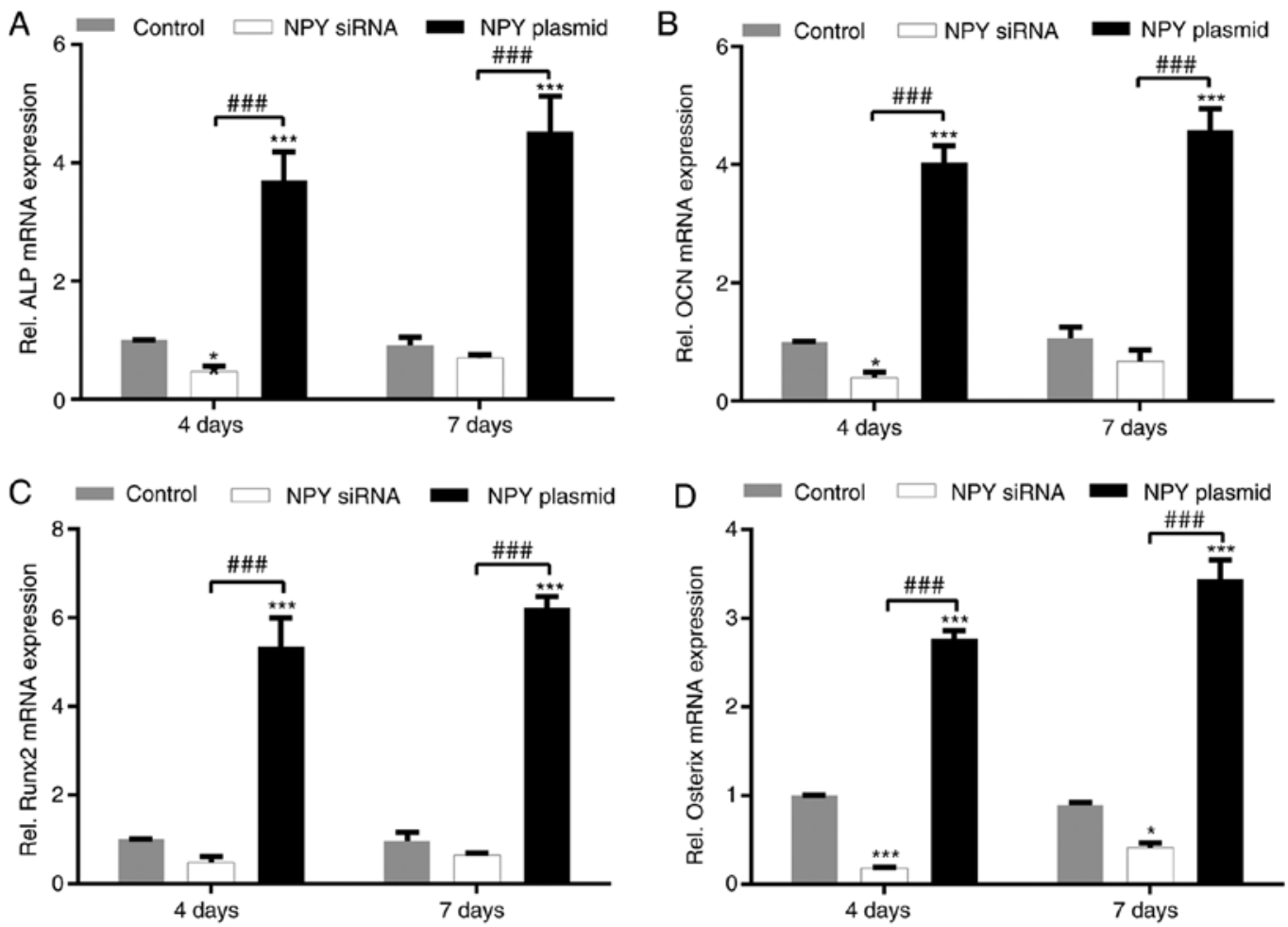

Figure 2. Expression of ALP, OCN, Runx2 and osterix mRNA in MC3T3-E1 cells. Expression of (A) ALP, (B) OCN, (C) Runx2 and (D) osterix mRNA at days 4 and $7 .{ }^{*} \mathrm{P}<0.05,{ }^{* * *} \mathrm{P}<0.001$ vs. control group; ${ }^{\# \# *} \mathrm{P}<0.001$ vs. siRNA group. NPY, neuropeptide $\mathrm{Y}$; ALP, alkaline phosphatase; OCN, osteocalcin; Runx2, runt-related transcription factor 2; siRNA, small interfering RNA.
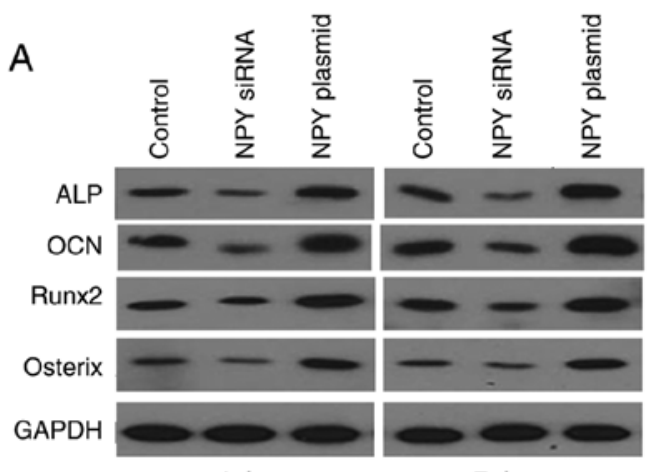

4 days

7 days
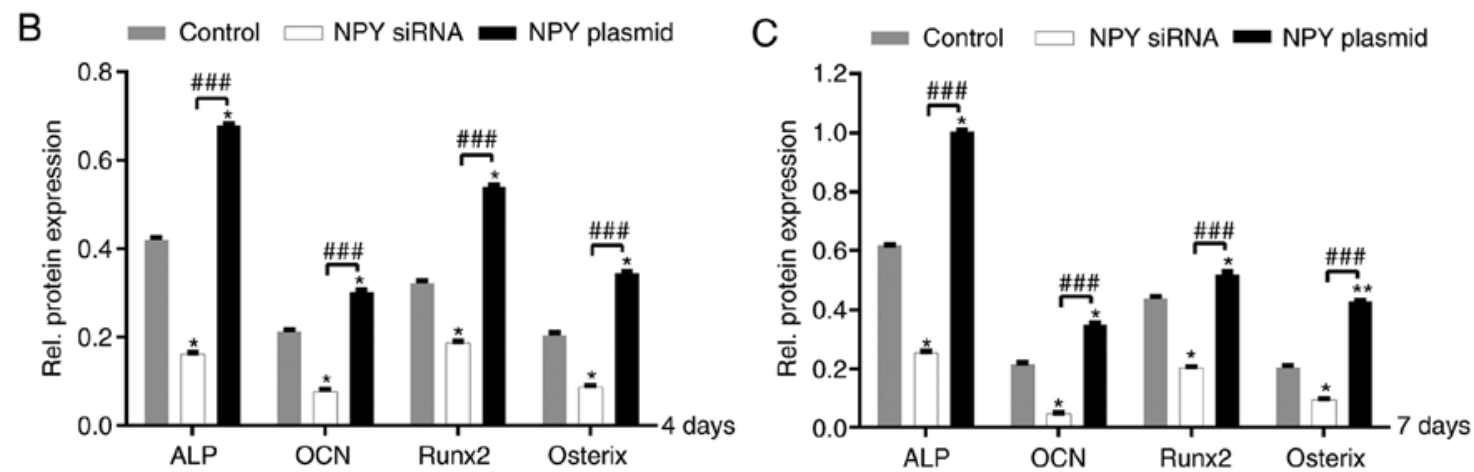

Figure 3. Expression of ALP, OCN, Runx2 and osterix protein in MC3T3-E1 cells. (A) Western blotting analysis of ALP, OCN, Runx2 and osterix protein expression at days 4 and 7. Semi-quantification of protein expression at (B) day 4 and (C) day $7 .{ }^{*} \mathrm{P}<0.05,{ }^{* * *} \mathrm{P}<0.01$ vs. control group; ${ }^{\# \# \#} \mathrm{P}<0.001$ vs. siRNA group. NPY, neuropeptide Y; siRNA, small interfering RNA; ALP, alkaline phosphatase; OCN, osteocalcin; Runx2, runt-related transcription factor 2.

osterix protein expression was decreased by $\sim 0.60$ - and 0.55 -fold at days 4 and 7 , respectively, compared with the control group $(\mathrm{P}<0.05$; Fig. 3A-C).
In conclusion, these findings indicated that NPY may be a positive regulator of osteogenic differentiation in mouse MC3T3-E1 cells. 


\section{Discussion}

NPY is a polypeptide of 36 amino acids, belonging to a larger family of neuropeptides that also includes the YY peptide and the pancreatic polypeptide $(21,22)$. Baldock et al (23) found that $\mathrm{Y} 1$ or $\mathrm{Y} 2$ receptor knockout in mice resulted in increased trabecular and cortical bone mass. However, within the bone microenvironment, the regulatory mechanisms of NPY at the genetic level remain unclear. As a result, the aim of the present study was to conduct an in-depth assessment of these hypotheses in the MC3T3-E1 cell line.

NPY is a neurotransmitter that has dual functions in the regulation of energy consumption and bone mass (11). A previous study determined that SNPs within NPY and neuraminidase (NA) were closely associated with the susceptibility and prognosis of patients with cervical vertigo (19). Therefore, NPY and NA could be used as diagnostic markers and therapeutic targets for cervical vertigo (19). Igwe et al (15) revealed that mouse calvarial osteoblasts fostered in the presence of NPY decreased the expression of differentiation-related markers, including OCN, BSP and dentin matrix acidic phosphoprotein 1 (DMP1), and also reduced the extent of mineralization. Their findings indicated that the levels of intracellular cAMP and differentiation markers (OCN, BSP and DMP1) in mouse calvarial osteoblasts were reduced by exogenous NPY treatment (15). In the present study, NPY was overexpressed in MC3T3-E1 cells to observe its effects on osteogenic ability. Overexpressing NPY was found to markedly enhance the osteogenic ability of mouse MC3T3-E1 cells via an autocrine mechanism, coincident with the upregulation of Runx 2 and osterix. In addition, the mRNA expression levels of ALP, OCN, osterix and Runx 2 were increased at different time points. In contrast to Igwe et al (15), the present study reported that endogenous NPY enhanced the expression of MC3T3-E1 cell differentiation markers, including ALP, OCN, Runx2 and osterix.

BMP signaling stimulates osteoblast differentiation and osteogenic activity by upregulating osterix and Runx2, which are essential osteogenic transcription factors $(20,24)$. Runx2 is an important factor for bone growth, and the heterozygous loss of Runx 2 results in clavicular dysplasia in humans, and the corresponding phenotype in mice (25). It has also been confirmed that homozygous Runx $2-\mathrm{II}^{-/-}$mice possess lower levels of bone mass and hypertrophic cartilage (25). Runx2-I is necessary for early bone growth and intramembranous ossification, whereas Runx2-II is needed for the complete maturation of osteoblasts and osteogenesis in cartilage (26). Osterix, a downstream effector of Runx 2 required for osteoblast differentiation, is significantly reduced in selective Runx2-II ${ }^{--}$mice (27). An associated decrease in the expression of bone ALP, OCN, osteopontin and matrix extracellular phosphoglycoprotein was also observed. However, the levels of these markers were normal or slightly decreased in heterozygous mutant mice (25). Despite this, the relationship between Runx 2 and osterix in osteogenic differentiation (in the MC3T3-E1 cell line specifically) remains to be elucidated. Nakashima et al (27) found that osterix was not expressed in Runx2-II ${ }^{--}$mice. In the present study, it was hypothesized that NPY may regulate osteoblast differentiation via the upregulation of Runx 2 and osterix, and the role of NPY in promoting the cell differentiation and maturation of MC3T3-E1 cells via the Runx 2 and osterix pathways was further validated. In a previous study based on the findings of Xiao et al (26), Runx2 was revealed to be the upstream regulatory gene of osterix. Runx2-I is also involved in maintaining $50 \%$ of osterix gene expression in Runx2-II-deficient (Runx2-II $\mathrm{II}^{-/}$) mice; thus, $50 \%$ of osterix mRNA may be regulated by upstream Runx 2 mRNA (26). In the present study (Fig. 2C and D), osterix gene expression was found to be consistent with that of Runx2, and the level of osterix gene expression was almost half that of Runx2. It was therefore hypothesized that osterix is a target gene of Runx2, by which it can be positively regulated.

The RFect siRNA and plasmid DNA transfection reagents were specifically designed by Changzhou Baidai Biotechnology Co., Ltd. (Patent application number: 20100022618) for transfecting siRNA and DNA into eukaryotic cells; this is a novel method derived from animal-origin free lipid transfection reagents, and the technique can be used on a wide range of adherent cell lines. It has numerous useful features; for example, the efficiency of transfection is $>90 \%$, and the effect of gene knockdown is markedly apparent (28). Furthermore, minimal cytotoxicity reduces non-specific effects and cellular stress $(28,29)$. Low concentrations of siRNA and DNA can be used to obtain high levels of knockdown and overexpression, respectively $(28,29)$. In the present study, the interference efficiency of NPY mRNA at day 4 reached $\sim 75$ and $80 \%$, respectively. In addition, the overexpression of NPY mRNA was $\sim 4$ - and 6-fold at days 4 and 7, respectively. However, it was also found that Runx2 gene and protein expression were not synchronized at the same time point. Following NPY knockdown, the expression of Runx 2 mRNA only showed a slight decrease, whereas the alteration in Runx2 protein expression was statistically significant. One reason could be that the expression of Runx 2 protein determined the behavior of the mouse MC3T3-E1 cells, whereas Runx 2 mRNA detection had temporal and spatial specificity, which may mean that the expression of Runx 2 mRNA and protein are not in sync at the same time point. This mechanism was also supported by previous articles $(24,25)$. Nevertheless, there were numerous limitations of the present study. One limitation is that the osteogenic differentiation was performed with the use of a single cell line for only 7 days, which also increases the uncertainty underlying the function of NPY in Runx2. Adding extra cell lines and prolonging the experiment time would increase the accuracy and validity of the experiment. Furthermore, the mechanism of action of NPY on Runx 2 is not clear, and further research is required in the future. Additional cell lines and more time points for osteogenic differentiation are needed in further experiments. However, overall, the RFect plasmid DNA and siRNA transfection technique, which was adopted in the present study, was found to be reliable for use with the MC3T3-E1 cell line.

Based on the results of previous studies (2,30-32), combining bioactive molecules, such as growth factors, peptides and small molecules with corresponding bone scaffold may lead to effective clinical transformation and provide new insights into the future of bone transplantation. Notably, this study demonstrated that NPY signaling directly promotes osteogenic differentiation of MC3T3-E1 cells by upregulating Runx2 and osterix in vitro. Additionally, NPY serves a positive role in bone formation, suggesting that NPY may be a therapeutic target for large bone defects caused by severe trauma, tumor resection, cancer or congenital diseases in the future. 


\section{Acknowledgements}

Not applicable.

\section{Funding}

The present study was supported by a grant from The Second Hospital, Cheeloo College of Medicine, Shandong University, P.R. China (grant no. 2018YT06).

\section{Availability of data and materials}

The datasets used and/or analyzed during the current study are available from the corresponding author on reasonable request.

\section{Authors' contributions}

BZ, XZha and CG designed the study. BZ, XZho and YC performed the experiments and collected the data. JX performed the statistical analysis. BZ and XZho wrote the manuscript. All authors read and approved the final manuscript.

\section{Ethics approval and consent to participate}

Not applicable.

\section{Patient consent for publication}

Not applicable.

\section{Competing interests}

The authors declare that they have no competing interests.

\section{References}

1. Yang F, Wang J, Hou J, Guo H and Liu C: Bone regeneration using cell-mediated responsive degradable PEG-based scaffolds incorporating with rhBMP-2. Biomaterials 34: 1514-1528, 2013.

2. Ho-Shui-Ling A, Bolander J, Rustom LE, Johnson AW, Luyten FP and Picart $\mathrm{C}$ : Bone regeneration strategies: Engineered scaffolds, bioactive molecules and stem cells current stage and future perspectives. Biomaterials 180: 143-162, 2018.

3. Baraban SC: Neuropeptide Y and limbic seizures. Rev Neurosci 9: 117-128, 1998.

4. Silva AP, Cavadas C and Grouzmann E: Neuropeptide Y and its receptors as potential therapeutic drug targets. Clin Chim Acta 326 3-25, 2002.

5. Baldock PA, Lin S, Zhang L, Karl T, Shi Y, Driessler F, Zengin A, Hörmer B, Lee NJ, Wong IP, et al: Neuropeptide Y attenuates stress-induced bone loss through suppression of noradrenaline circuits. J Bone Miner Res 29: 2238-2249, 2014.

6. KuoLE,KitlinskaJB,Tilan JU,LiL,BakerSB,Johnson MD,LeeEW, Burnett MS, Fricke ST, Kvetnansky R, et al: Neuropeptide Y acts directly in the periphery on fat tissue and mediates stress-induced obesity and metabolic syndrome. Nat Med 13: 803-811, 2007.

7. Baldock PA, Allison SJ, Lundberg P, Lee NJ, Slack K, Lin EJD, Enriquez RF, McDonald MM, Zhang L, During MJ, et al: Novel role of $\mathrm{Y} 1$ receptors in the coordinated regulation of bone and energy homeostasis. J Biol Chem 282: 19092-19102, 2007.

8. Yang K, Guan H, Arany E, Hill DJ and Cao X: Neuropeptide Y is produced in visceral adipose tissue and promotes proliferation of adipocyte precursor cells via the Y1 receptor. FASEB J 22: 2452-2464, 2008

9. Khor EC and Baldock P: The NPY system and its neural and neuroendocrine regulation of bone. Curr Osteoporos Rep 10: 160-168, 2012 .
10. Horsnell $\mathrm{H}$ and Baldock PA: Osteoblastic actions of the neuropeptide $\mathrm{Y}$ system to regulate bone and energy homeostasis. Curr Osteoporos Rep 14: 26-31, 2016.

11. Lin S, Boey D and Herzog H: NPY and Y receptors: Lessons from transgenic and knockout models. Neuropeptides 38: 189-200, 2004.

12. Allison SJ, Baldock PA and Herzog H: The control of bone remodeling by neuropeptide Y receptors. Peptides 28: 320-325, 2007.

13. Yahara $\mathbf{M}$, Tei $\mathrm{K}$ and Tamura $\mathrm{M}$ : Inhibition of neuropeptide $\mathrm{Y}$ Y1 receptor induces osteoblast differentiation in MC3T3E1 cells. Mol Med Rep 16: 2779-2784, 2017.

14. Karsenty G: Transcriptional control of skeletogenesis. Annu Rev Genomics Hum Genet 9: 183-196, 2008.

15. Igwe JC, Jiang X, Paic F, Ma L, Adams DJ, Baldock PA, Pilbeam CC and Kalajzic I: Neuropeptide Y is expressed by osteocytes and can inhibit osteoblastic activity. J Cell Biochem 108: 621-630, 2009.

16. Sudo H, Kodama HA, Amagai Y, Yamamoto S and Kasai S: In vitro differentiation and calcification in a new clonal osteogenic cell line derived from newborn mouse calvaria. J Cell Biol 96: 191-198, 1983.

17. Livak KJ and Schmittgen TD: Analysis of relative gene expression data using real-time quantitative PCR and the 2(-Delta Delta C(T)) method. Methods 25: 402-408, 2001.

18. Chen J, He G, Wang Y and Cai D: MicroRNA-223 promotes osteoblast differentiation of MC3T3-E1 cells by targeting histone deacetylase 2. Int J Mol Med 43: 1513-1521, 2019.

19. Han J, Zuo J, Zhu D and Gao C: The correlation between SNPs within the gene of adrenergic receptor and neuropeptide $\mathrm{Y}$ and risk of cervical vertigo. J Clin Lab Anal 32: e22366, 2018.

20. Li L, Sapkota M, Gao M, Choi H and Soh Y: Macrolactin F inhibits RANKL-mediated osteoclastogenesis by suppressing Akt, MAPK and NFATc1 pathways and promotes osteoblastogenesis through a BMP-2/smad/Akt/Runx2 signaling pathway. Eur J Pharmacol 815: 202-209, 2017.

21. Tatemoto K, Carlquist M and Mutt V: Neuropeptide Y-a novel brain peptide with structural similarities to peptide YY and pancreatic polypeptide. Nature 296: 659-660, 1982.

22. Allen JM, Adrian TE, Tatemoto K, Polak JM, Hughes J and Bloom SR: Two novel related peptides, neuropeptide Y (NPY) and peptide YY (PYY) inhibit the contraction of the electrically stimulated mouse vas deferens. Neuropeptides 3: 71-77, 1982.

23. Baldock PA, Sainsbury A, Allison S, Lin EJ, Couzens M, Boey D, Enriquez R, During M, Herzog H and Gardiner EM: Hypothalamic control of bone formation: distinct actions of leptin and $\mathrm{y} 2$ receptor pathways. J Bone Miner Res 20: 1851-1857, 2005.

24. Moon JS, Kim SH, Oh SH, Jeong YW, Kang JH, Park JC, Son HJ, Bae S, Park BI, Kim MS, et al: Relaxin augments BMP-2-induced osteoblast differentiation and bone formation. J Bone Miner Res 29: 1586-1596, 2014.

25. Hecht J, Seitz V, Urban M, Wagner F, Robinson PN, Stiege A, Dieterich C, Kornak U, Wilkening U, Brieske N, et al: Detection of novel skeletogenesis target genes by comprehensive analysis of a Runx2(-/-) mouse model. Gene Expr Patterns 7: 102-112, 2007.

26. Xiao ZS, Hjelmeland AB and Quarles LD: Selective deficiency of the 'bone-related' Runx2-II unexpectedly preserves osteoblast-mediated skeletogenesis. J Biol Chem 279: 20307-20313, 2004.

27. Nakashima K, Zhou X, Kunkel G, Zhang Z, Deng JM, Behringer RR and de Crombrugghe $\mathrm{B}$ : The novel zinc finger-containing transcription factor osterix is required for osteoblast differentiation and bone formation. Cell 108: 17-29, 2002.

28. Yang X, Yang Y, Sun BF, Chen YS, Xu JW, Lai WY, Li A, Wang X, Bhattarai DP, Xiaot W, et al: 5-methylcytosine promotes mRNA export-NSUN2 as the methyltransferase and ALYREF as an $\mathrm{m}^{5} \mathrm{C}$ reader. Cell Res 27: 606-625, 2017.

29. Liu J, Fang H, Chi Z, Wu Z, Wei D, Mo D, Niu K, Balajee AS, Hei TK, Nie L and Zhao Y: XPD localizes in mitochondria and protects the mitochondrial genome from oxidative DNA damage. Nucleic Acids Res 43: 5476-5488, 2015.

30. Gao C, Peng S, Feng P and Shuai C: Bone biomaterials and interactions with stem cells. Bone Res 5: 17059, 2017.

31. Shuai C, Xu Y, Feng P, Wang G, Xiong S and Peng S: Antibacterial polymer scaffold based on mesoporous bioactive glass loaded with in situ grown silver. Chemical Engineering J 374: 304-315, 2019.

32. Yang Y, Zan J, Yang W, Qi F, He C, Huang S, Peng S and Shuai C: Metal organic frameworks as a compatible reinforcement in a biopolymer bone scaffold. Materials Chem Front 4: 973-984, 2020.

(i) $\Theta$ This work is licensed under a Creative Commons Attribution-NonCommercial-NoDerivatives 4.0 International (CC BY-NC-ND 4.0) License. 